\title{
THE
}

UNIVERSITY

University of Rhode Island

OF RHODE ISLAND

DigitalCommons@URI

7-11-1994

\section{Transport Phenomena at Rough Boundaries}

\author{
A. E. Meyerovich \\ University of Rhode Island, sfo101@uri.edu \\ S. Stepaniants \\ University of Rhode Island
}

Follow this and additional works at: https://digitalcommons.uri.edu/phys_facpubs

Terms of Use

All rights reserved under copyright.

\section{Citation/Publisher Attribution}

Meyerovich, A. E., \&.Stepaniants, S. (1994). Transport Phenomena at Rough Boundaries. Phys. Rev. Lett., 73(2), 316-319. doi: 10.1103/PhysRevLett.73.316

Available at: http://dx.doi.org/10.1103/PhysRevLett.73.316

This Article is brought to you for free and open access by the Physics at DigitalCommons@URI. It has been accepted for inclusion in Physics Faculty Publications by an authorized administrator of DigitalCommons@URI. For more information, please contact digitalcommons-group@uri.edu. 


\title{
Transport Phenomena at Rough Boundaries
}

\author{
A. E. Meyerovich and S. Stepaniants \\ Department of Physics, University of Rhode Island, Kingston, Rhode Island 02881
}

(Received 10 March 1994)

\begin{abstract}
We present a simple method describing transport processes near rough boundaries. By a proper coordinate transformation we reduce a transport problem with rough walls to an equivalent problem with smooth flat walls, but with some random bulk inhomogeneities. In many cases the last problem can be treated perturbatively, leading to simple expressions for relevant transport coefficients via the correlation function of surface inhomogeneities. We calculate diffusion and conductivity in films, phonon, and photon diffusion, quantum corrections to conductivity, and the single-particle diffusion coefficient.
\end{abstract}

PACS numbers: 72.10.Bg, 73.50.Bk, 79.20.Rf

The study of transport processes near the boundaries is important for all branches of physics. The roughness of boundaries with random inhomogeneities of different scales leads to a considerable complication. The scattering by rough walls is described either by a complicated exact boundary condition (see, e.g., Refs. [1-5]) which leads to a practically unsolved integrodifferential transport problem, or by an oversimplified condition with some balance between specular and diffuse reflection. The perturbative methods [4] have a rather limited applicability. Below we demonstrate an alternative general description of the effect of surface inhomogeneities on transport near the surface.

The problem of scattering of particles by rough walls has two parts. The energy spectrum, including the potential relief and energy bands, changes near the walls; this effect exists for perfect walls. Roughness of the wall, i.e., randomness in the exact position and the direction of the boundary, leads to randomness in phases and directions of reflected particles even in the absence of energy changes. These two groups of effects can often be separated.

In many metals or semiconductors, or in systems with boundary adsorption, the particle energy experiences dramatic changes near the surface; then such changes dominate the scattering. In many problems of wave propagation, gas dynamics, electrons in simple metals, etc., the change of energy near the surface plays the secondary role with respect to the effects of surface roughness. Then the distortion of energy near the walls can be addressed after the effects of surface roughness are well understood. We are interested in this class of problem.

The boundary roughness causes an additional diffusion along the boundaries. The problem is how to express this extra chaotic motion by the boundaries via the correlation function of surface inhomogeneities. Usually, the solutions are different for different classes of systems.

Below we present a simple and versatile approach to transport problems near the rough walls. Originally we developed this method for the particular case of transverse spin diffusion in spin-polarized quantum gases [6].
It turned out that this method is very general, and is useful for a wide variety of transport problems. We will apply it to diffusion and conductivity of electrons, diffusion of phonons and photons, calculation of quantum (localization) corrections for conductivity, and the classical problem of chaotic motion of a bouncing ball (i.e. singleparticle diffusion). In all these, and many other cases, the effects of boundary can be described in a uniform way. Another advantage is a relative simplicity of calculations.

The idea is to perform a coordinate transformation (similar to the Migdal transformation in nuclear physics) so as to make the walls flat. This is possible if the boundaries have no cavities and are described by single-valued functions. As a result of this nonlinear canonical transformation, the bulk Hamiltonian acquires additional random terms which depend on initial boundary roughness. If the amplitude of the boundary roughness is not large, these corrections to the bulk Hamiltonian can be treated within the perturbative transport equation leading to transparent expressions for the transport coefficients. For simplicity, we neglect all bulk scattering processes, and consider the scattering by random surface inhomogeneities as a sole source of chaos and formation of the mean free path.

We consider a film of average thickness $L$ with the boundaries $x=L / 2-\xi_{1}(y, z)$ and $x=-L / 2+\xi_{2}(y, z)$. [For 2D problems one should disregard $z$.] The inhomogeneities are small, $\xi_{1} \xi_{2} \ll L$, and random, $\left\langle\xi_{1}\right\rangle=\left\langle\xi_{2}\right\rangle=$ 0 . The results should be expressed via the binary correlation functions $\zeta_{i k}\left(\left|\mathbf{s}_{1}-\mathbf{s}_{2}\right|\right)=\left\langle\xi_{i}\left(\mathbf{s}_{1}\right) \xi_{k}\left(\mathbf{s}_{2}\right)\right\rangle$ which depends only on the distance between $\mathbf{s}_{1}$ and $\mathbf{s}_{2}$ (usually, the inhomogeneities from different boundaries are uncorrelated, $\zeta_{12}=0$ ). For simplicity, we consider the impenetrable walls with the boundary condition $\Psi=0$. The shift of inhomogeneity from the boundary to the bulk is achieved by the coordinate transformation similar to [6]

$$
X=\frac{L\left\{x-\frac{1}{2}\left[\xi_{2}(y, z)-\xi_{1}(y, z)\right]\right\}}{L-\left[\xi_{1}(y, z)+\xi_{2}(y, z)\right]}, \quad Y=y, \quad Z=z .
$$

This transformation makes both boundaries flat $X=$ $\pm L / 2$. 
Canonic transformation (1) requires a conjugate transformation of momenta $\mathbf{p} \rightarrow \mathbf{P}$. The bulk Hamiltonian should be expressed through the new variables $\{\mathbf{R}, \mathbf{P}\}$. If the initial Hamiltonian is $\hat{H}=p^{2} / 2 m$, then in variables $\{\mathbf{R}, \mathbf{P}\}$ it obtains random "perturbation" $\hat{V},\langle\hat{V}\rangle=0$, which, after expansion in $\xi / L$, has the form

$$
\begin{gathered}
\hat{H}=\hat{P}^{2} / 2 m+\hat{V}, \\
\hat{V}=\frac{1}{2 m}\left(\frac{2 \xi}{L} \hat{P}_{x}^{2}+X \hat{P}_{x} \frac{\xi_{y}^{\prime}}{L} \hat{P}_{y}+X \hat{P}_{x} \frac{\xi_{z}^{\prime}}{L} \hat{P}_{z}+\text { H.c. }\right)^{(2)},
\end{gathered}
$$

where $\xi \equiv \xi_{1}+\xi_{2}$. As a result, the problem of transport within a system with rough walls is reduced to a completely equivalent problem of transport in a system with ideal smooth walls, $\Psi(L / 2)=\Psi(-L / 2)=0$, but with the perturbed bulk Hamiltonian (2).

In thin films the transverse motion is quantized with distinct quantum numbers $n$. In thick films (classical or WKB transverse motion) these quantum numbers are large, and this quantization is irrelevant. The following calculations can be easily performed for both cases; for brevity, we present the results, except for the density of states in thin 2D films, only for the cases when one may neglect the transverse quantization.

The transport coefficient should be calculated from the transport equation. The effective collision integral on the right-hand side of the transport equation for particles contains the transition probabilities between different states:

$$
L_{\mathrm{coll}}=\int W\left(\mathbf{P}, \mathbf{P}^{\prime}\right)\left(n^{\prime}-n\right) V d^{3} P^{\prime} /(2 \pi \hbar)^{3}
$$

[ $n(\mathbf{p})$ and $n^{\prime}=n\left(\mathbf{p}^{\prime}\right)$ are the distribution functions, $V$ is the volume]. The transition probability is the square of the matrix element of the bulk perturbation (2) multiplied by the $\delta$ function of energies

$$
W\left(\mathbf{P}, \mathbf{P}^{\prime}\right)=(2 \pi / \hbar)\left|V_{q P_{x}, q^{\prime} P_{x}^{\prime}}\right|^{2} \delta\left(\epsilon-\epsilon^{\prime}\right)
$$

and, after averaging, becomes proportional to Fourier image $\zeta(\mathbf{q})$ of the correlation function of surface inhomogeneities $\zeta\left(\left|\mathbf{s}_{1}-\mathbf{s}_{2}\right|\right)=\left\langle\xi\left(\mathbf{s}_{1}\right) \xi\left(\mathbf{s}_{2}\right)\right\rangle\left(\mathbf{q} \equiv \mathbf{P}_{\perp}\right.$ is the component of momentum along the walls).

Other transport problems, e.g., the single-particle diffusion between two rough walls, require the use of the Fokker-Plank equation instead of the Boltzmann equation. The tensor of diffusion coefficients in momentum space in the Fokker-Plank equation is equal to $\left(\mathbf{Q}=\mathbf{P}-\mathbf{P}^{\prime}\right)$

$$
D_{i k}^{(p)}=\frac{1}{2} \int Q_{i} Q_{k} W\left(\mathbf{P}, \mathbf{P}^{\prime}\right) d^{3} Q .
$$

The electrical conductivity is determined by the linearized Boltzmann equation which can be solved at arbitrary degree of quantum degeneracy of the (electron) system:

$$
\sigma=-\frac{4}{\pi} e^{2} L^{2} \int \frac{\partial n_{0}}{\partial \epsilon} \frac{(2 m \epsilon)^{-1 / 2}}{\left(\zeta_{0}-\zeta_{1}\right)} \frac{d \epsilon}{\cos ^{2} \theta} \frac{d \cos \theta}{\alpha+4 \tan ^{4} \theta},
$$

where $\theta$ is the angle between $\mathbf{P}$ and the plane of the wall, $n_{0}(\epsilon)$ is the equilibrium distribution, $\zeta_{i}, \eta_{i}$ are the Fourier harmonics of the functions $\zeta, \eta$ over the angle $\phi$, and

$$
\begin{gathered}
\alpha=\left(\eta_{0}-\eta_{1}\right) /\left(\zeta_{0}-\zeta_{1}\right), \\
\eta(P, \theta, \phi)=\zeta(P \cos \theta, \phi)[1-\cos \phi]^{2} .
\end{gathered}
$$

The 0th Fourier harmonic of the correlation function of surface inhomogeneities, $\zeta(\phi)[1-\cos \phi]$, plays the role of the transport cross section for particles. For degenerate electrons at $T \rightarrow 0$,

$$
\begin{gathered}
\sigma=\frac{4}{\pi} \frac{e^{2} L^{2}}{p_{F}} \int \frac{1}{\zeta_{0}-\zeta_{1}} \frac{1}{\cos ^{2} \theta} \frac{d \cos \theta}{\alpha+4 \tan ^{4} \theta}, \\
\zeta=\zeta\left(p_{F} \cos \theta, \phi\right)
\end{gathered}
$$

while at high temperatures,

$$
\begin{gathered}
\sigma=8 \pi \frac{e^{2} L^{2} \hbar^{3}}{m^{2} T^{2}} \int \frac{\exp (-x)}{\zeta_{0}-\zeta_{1}} \frac{x^{-1 / 2} d x}{\cos ^{2} \theta} \frac{d \cos \theta}{\alpha+4 \tan ^{4} \theta}, \\
\zeta=\zeta\left((2 m x)^{1 / 2} \cos \theta, \phi\right) .
\end{gathered}
$$

The diffusion coefficient differs from $\sigma$ by the density of states. Only the diagonal diffusion coefficients $D_{y y}$ and $D_{z z}$ are nonzero:

$$
D_{y y}=D_{z z}=\frac{\pi^{2} \hbar^{3} \sigma}{2 m e^{2}} / \int \frac{\partial n_{0}}{\partial \epsilon}(2 m \epsilon)^{1 / 2} d \epsilon .
$$

By the order of magnitude,

$$
D \sim(\hbar / m) x^{-2 / 3}(a L / l R)^{2}\left(T_{F} / E\right),
$$

where $x=a^{3} N$ is the atomic (dimensionless) density of the gas between the walls, $l^{2}=\zeta(0)$ and $R$ are the average height and correlation radius of surface inhomogeneities, and the characteristic energy $E$ is the larger of temperature $T$ and the bulk degeneracy temperature $T_{F}$. This should be compared with the standard bulk diffusion with the particle cross section $\sigma$ :

$$
D_{0} \sim(\hbar / m) x^{-2 / 3}\left(a^{2} / \sigma\right)\left(T_{F} / E\right)^{-1 / 2}(E / T)^{2} .
$$

A different problem is diffusion (Brownian) motion of a single particle between random rough walls (a classical "bouncing ball"). This is equivalent to the diffusion problem for a particle with random bulk Hamiltonian (2) between specular flat walls. Some distinct features of the Hamiltonian (2) (e.g., the momentum dependence of the random "perturbation" $\hat{V}$ ) make it different from random Hamiltonians used in typical diffusion problems. It may well be that these particular features are responsible for some anomalies of motion of particles between random walls which were observed in computations. We will not discuss this problem here, and will assume that the motion 
is diffusive. Then the single-particle diffusion coefficient can be trivially derived from the diffusion coefficient in the momentum space $\overleftrightarrow{D^{(p)}}$. This coefficient depends on the energy of a particle $\epsilon$ ( $\epsilon$ is an integral of motion), and the average $x$ component of the momentum $P_{x}$. The diffusion time is determined by $\overleftrightarrow{D^{(p)}}$ as the time in which the component of momentum along the walls changes from $\mathbf{q}$ to $-\mathbf{q}$. The single-particle diffusion coefficient resembles the integrand in $D$ and $\sigma$, Eq. (3):

$$
D_{y y}=D_{z z}=\frac{2 \pi \hbar^{3} L^{2}}{\left(\zeta_{0}-\zeta_{1}\right)} \frac{\epsilon-P_{x}^{2} / 2 m}{\alpha\left(2 m \epsilon-P_{x}^{2}\right)^{2} / 4+P_{x}^{4}} .
$$

Even if the motion of a bouncing ball does not have diffusive asymptotics, our approach may be very convenient for verification of computational predictions: A numerical study of particle with a random bulk Hamiltonian is easier and more straightforward than a study of reflections from random walls. In classical mechanics, the Hamiltonian (2) is equivalent to a particle with a random coordinate-dependent anisotropic effective mass,

$$
\begin{gathered}
m_{x x}^{*-1}=(1+2 \xi / L) / m, \\
m_{x y}^{*-1}=X \xi_{y}^{\prime} / m L, \quad m_{x z}^{*-1}=X \xi_{z}^{\prime} / m L
\end{gathered}
$$

(the rest of the components do not change).

Above we evaluated the mean free path formed by collisions with random rough walls. This allows us to calculate the quantum (localization) corrections to conductivity associated with the walls. In a 3D case [7],

$$
\frac{\Delta \sigma}{\sigma}=-\int_{\tau}^{\tau_{\theta}} v \lambda^{2}\left(D_{y y} t\right)^{-3 / 2} d t=-\frac{8 \pi^{2} \hbar^{2}}{m^{2} D_{y y}^{2}}
$$

( $\lambda$ is the de Broglie wavelength; in the last equality the phase relaxation time $\tau_{\phi} \rightarrow \infty$ ).

Quantum corrections for a 2D film with random linear boundaries are different. We can calculate the localization gap and the density of states when the distance between the boundaries is so small that transitions between quantized levels $P_{x}=\pi n \hbar / L$ for transverse motion are impossible. For $\delta$-correlated boundary inhomogeneities, $\left\langle\xi\left(y_{1}\right) \xi\left(y_{2}\right)\right\rangle=\left(\hbar^{4} L^{* 3} / m^{2} L^{6}\right) \delta\left(y_{1}-y_{2}\right)$, the density of states for the motion in the $y$ directions is

$$
\begin{gathered}
{[8]\left[\left(L^{*}\right)^{3}=n^{4} l^{2} R\right]} \\
v^{-1}(E)=\frac{\pi^{1 / 2} L^{*}}{2^{1 / 2} L^{2}} \int_{0}^{\infty} \frac{d z}{\sqrt{z}} \exp \left(-\frac{z^{3}}{24}-z \mathcal{E}\right), \\
\mathcal{E}=\frac{E m L^{2}}{\hbar^{2}} \frac{L^{2}}{L^{* 2}} .
\end{gathered}
$$

Up to now, we considered particles with quadratic energy spectrum, $p^{2} / 2 m$. The results can be extended to arbitrary spectra, $\epsilon(\mathbf{p})$, e.g., "relativistic" particles (phonons or phonons) with the spectrum $\epsilon=c p$. After the transformation (1), this Hamiltonian obtains the form

$$
\begin{gathered}
\hat{H}_{1}=c \sqrt{2 m(\hat{H}+\hat{V})} \simeq c\left(2 m \hat{H}_{0}\right)^{1 / 2}+\delta \hat{H}_{1}, \\
\delta \hat{H}_{1}=c(m / 2)^{1 / 2} \hat{H}_{0}^{-1 / 2} \hat{V},
\end{gathered}
$$

with $\hat{H}_{0}$ and $\hat{V}$ from (2). Since the plane waves are the eigenstates of both operators $\hat{H}_{0}$ and unperturbed $\hat{H}_{1}$, the square of the matrix elements of the "perturbation" $\delta \hat{H}_{1}$ in the scattering probability $W\left(\mathbf{P}, \mathbf{P}^{\prime}\right)$ differs from $\left|V_{\mathbf{P P}}\right|^{2}$ only by the factor $c^{2} m^{2} / P^{2}$. The diffusion coefficient is

$$
D_{y y}=D_{z z}=\frac{\pi \hbar^{3} c^{2} L^{2}}{P^{2}\left(\zeta_{0}-\zeta_{1}\right)} \frac{\epsilon^{2} / c^{2}-P_{x}^{2}}{\alpha\left(\epsilon^{2} / c^{2}-P_{x}^{2}\right)^{2} / 4+P_{x}^{4}} .
$$

In summary, we calculated transport coefficients for different quantum and classical problems of particle and wave transport near the rough walls. All transport coefficients are expressed via the correlation function of surface inhomogeneities. The method is astonishingly simple computationally and is very versatile. Note that the transport coefficients contain not only the correlation of inhomogeneities from the same wall, but also the correlation of inhomogeneities from the opposite walls. This correlation is important only as far as we neglect the bulk relaxation processes, and should disappear from the expressions with an increase in bulk relaxation.

The incorporation of bulk relaxation is an obvious way to continue this work. We can also include the change of the potential relief near the walls, $U\left(x-L / 2+\xi_{1}\right)$ and $U\left(x+L / 2-\xi_{2}\right)$, into the Hamiltonian. This will add to the Hamiltonian (2) regular terms, $U(X \mp L / 2)$, and small random parts, $\delta U\left(X, \xi_{1} / L, \xi_{2} / L\right)$. The regular part will change the wave functions for calculation of matrix elements, while the random part $\delta U$ should be treated in the same way as and simultaneously with the perturbation $V(2)$. The procedure and the implications for the results are obvious.

One more extension is the modification of the boundary condition $\Psi=0$ (infinite boundary barriers for particles or waves). The results for finite barriers will describe the diffusion in layered media and the transmission and reflection coefficients for layers with rough walls.

An interesting application can be the effect of surface roughness on the boundary slip. There are experimental and theoretical indications [9] that part of the boundary slip is caused by and has the scale of the boundary roughness. Our method can express this part of the slip length via the correlation function of boundary roughness.

All these extensions are rather straightforward. A nontrivial application of our method is the problem of the onset of chaos in the motion of a classical particle which is repeatedly reflected by rough walls (a "bouncing ball"). We discussed this problem assuming the diffusive character of the particle motion. There are 
some numerical indications that the asymptotic motion of a ball deviates from the diffusion behavior $l \propto D t^{2}$. It is not clear whether this deviation is an intrinsic property of the system, or a result of insufficient accuracy of computations. Our method can answer this question by reducing the computational problem of scattering by a random wall to an equivalent, but much more transparent, bulk computational problem. If the deviation from a diffusive asymptotic persists, it will mean that either the Hamiltonian (2) has some very special properties separating it from other random potentials, or that the diffusion chaotic motion in a random bulk potential is more complicated than is usually assumed.

Details of the calculations will be published elsewhere. This work was supported by NSF Grant No. DMR9100197.

[1] L. A. Falkovskii, Sov. Phys. JETP 31, 981 (1970).

[2] J. A. DeSanto and G.S. Brown, in Analytical Techniques for Multiple Scattering from Rough Surfaces, Progress in
Optics, Vol. XXIII, edited by E. Wolf (North-Holland, Amsterdam, 1986).

[3] J.R. Willis, in Mathematic Modeling in Non-Destructive Testing, edited by M. Blakemore and G. A. Georgiou, (Clarendon, Oxford, 1988), p. 57.

[4]. J. A. Ogilvy, Theory of Wave Scattering from Random Surfaces, (Adam Hilger, Bristol, 1991).

[5] Interactions of Atoms and Molecules with Solid Surfaces, edited by V. Bortolani, N. H. March, and M.P. Tosi (Plenum, New York, 1990).

[6] A.E. Meyerovich and S. Stepaniants, Phys. Rev. B 49, 3400 (1994).

[7] B. Altshuler, in Nanostructures and Mesoscopic Systems, edited by W. P. Kirk and M. A. Reed (Academic, New York, 1991), pp. 405-416.

[8] C. Itzykson and J.-M. Drouffe, Statistical Field Theory (Cambridge Univ. Press, Cambridge, 1991), Vol. 2, Chap. 10.

[9] D. A. Ritchie et al., Phys. Rev. Lett. 59, 465 (1987); D. Einzel, P. Panzer, and M. Liu, Phys. Rev. Lett. 64, 2269 (1990). 